\title{
Therapeutic Hypothermia Improves Long-Term Survival and Blunts Inflammation in Rats During Resuscitation of Hemorrhagic Shock
}

\author{
Jianru Shi, PhD, ${ }^{1,2}$ Wangde Dai, MD, ${ }^{1,2}$ Juan Carreno, RLATG, CMAR, ${ }^{1}$ \\ Lifu Zhao, MD, and Robert A. Kloner, MD, PhD ${ }^{1,2}$
}

\begin{abstract}
We tested the hypothesis that therapeutic hypothermia (TH) improves survival and blunts inflammation in rats undergoing experimental hemorrhagic shock. Rats were randomized to $\mathrm{TH}(n=16)$ or normothermia $(n=15)$. Hemorrhagic shock was induced by withdrawing blood to a fixed mean blood pressure (MBP) of $30 \mathrm{mmHg}$ for 30 minutes followed by reinfusion of shed blood for the next 30 minutes. TH (target $32^{\circ} \mathrm{C}$ ) was started at 5 minutes after MBP reached $30 \mathrm{mmHg}$ and was maintained throughout blood volume resuscitation. In the normothermic control group, body temperature was maintained at $37^{\circ} \mathrm{C}$ during the procedure. Rats were allowed to recover for 6 weeks. TH significantly improved survival: 4 of $15(26.7 \%)$ rats in the normothermic group and 11 of $16(68.8 \% ; p=0.032)$ rats in the TH group survived 6 weeks. Recovery of MBP during the resuscitation phase was significantly improved and left ventricular fractional shortening was markedly increased in the $\mathrm{TH}$ group compared with the normothermic group. Brain infarction was observed in 3 of 4 surviving rats (75\%) in normothermic group, and in only 1 of the 11 surviving rats (9\%) in TH group. The neutrophil-to-lymphocyte ratio was lower in TH group $(0.20 \pm 0.02)$ compared with the normothermic group $(0.32 \pm 0.03 ; p=0.003)$. TH influenced the levels of blood gases and blood counts, favoring hypothermia over control. TH significantly improved long-term survival and blunted the inflammatory response in experimental hemorrhagic shock.
\end{abstract}

Keywords: hypothermia, hemorrhagic shock, survival, inflammation

\section{Introduction}

$\mathbf{H}$ EMORRHAGIC SHOCK REMAINS a major cause of mortality and morbidity among soldiers on the battlefield and among civilians who are accident victims (Kalkwarf and Cotton, 2017). Even with restoration of blood volume, organs subjected to hemorrhagic shock can develop ischemia/ reperfusion injury and fail. The majority of battlefield casualties die before reaching the hospital and the most likely cause of death is hemorrhage (Hooper et al., 2014; Eastridge et al., 2019). Therefore, innovative approaches are needed to improve survival and protect vital organs during and after hemorrhagic shock.

Therapeutic hypothermia (TH) is known to protect vital organs in some cases of injury from ischemia/reperfusion. Several laboratory studies have suggested that $\mathrm{TH}$ provides benefits in models of hemorrhagic shock (Prueckner et al., 2001; Takasu et al., 2000, 2003, 2006). However, the potentially deleterious effects of hypothermia in the context of hemorrhagic shock are still a matter of debate. Studies from our research group demonstrated that TH profoundly reduced the size of experimentally induced acute myocardial infarction (MI) (Hale and Kloner, 1997, 2011; Herring et al., 2014). In two separate experimental models, $\mathrm{TH}$ administered during the phase of ischemia reduced the size of MI to the greatest extent: in rabbits $\mathrm{TH}$ reduced the size of the MI by $82 \%$ and in rats by $73 \%$ (Herring et al., 2015). We have recently demonstrated that the transient administration of $\mathrm{TH}$ during acute MI preserved myocardium long term, resulting in a smaller scar, better preservation of left ventricular structure with less dilatation and left ventricular remodeling and markedly improved left ventricular ejection fraction compared with normothermia (Dai et al., 2015).

MI is a local ischemic insult, whereas hemorrhagic shock is characterized by a state of total body (global) ischemia. These two conditions share similar mechanisms of injury. The injury (local or global) results from loss of blood and lack of perfusion of oxygen and nutrients to the organs; the tissue becomes acidotic and the cells remain hypoxic and at some point even if blood is restored the cells are irreversibly

\footnotetext{
${ }^{1}$ HMRI Cardiovascular Research Institute, Huntington Medical Research Institutes, Pasadena, California, USA.

${ }^{2}$ Division of Cardiovascular Medicine of the Keck School of Medicine, University of Southern California, Los Angeles, California, USA.
} 
injured, leading to failure of the vital organ. In addition, reoxygenation of organs that have been deprived of oxygen may cause generation of toxic reactive oxygen species and stimulate inflammatory reactions, which may themselves further damage tissue (reperfusion injury) (Kalogeris et al., 2012).

Therefore, we hypothesize that $\mathrm{TH}$, a therapy that has been shown to be cardioprotective and limit myocardial infarct size and improve cardiac function in the setting of infarction, could be applied as an acute therapy to improve survival and protect vital organs subjected to ischemia/reperfusion due to hemorrhagic shock.

\section{Methods}

This study was approved by the Institutional Animal Care and Use Committee at Huntington Medical Research Institutes and was performed in accordance with the Guidelines for the Care and Use of Laboratory Animals (NIH publication No. 85-23, National Academy Press, Washington DC, revised 2011).

\section{Hemorrhagic shock model}

Details of these methods have been published previously (Dai et al., 2019). In brief, age-matched (8 weeks old) Sprague-Dawley rats (both sexes), obtained from Charles River, Inc., were anesthetized with intraperitoneal ketamine $(90 \mathrm{mg} / \mathrm{kg})$ and xylazine $(10 \mathrm{mg} / \mathrm{kg})$. A cannula was inserted into the trachea and attached to a respirator; the rats were ventilated with room air at 60 strokes $/ \mathrm{min}$ and $10 \mathrm{~mL} / \mathrm{kg}$ tidal volume. Body temperature was maintained at $37^{\circ} \mathrm{C}$ with a heating pad. Under aseptic conditions, the left femoral artery, carotid artery, and jugular vein were dissected using a minimal dissection technique, distally ligated, and cannulated with a polyethylene catheter (PE-50) filled with heparinized saline $(8 \mathrm{IU} / \mathrm{mL})$ to avoid local coagulation. The femoral artery catheter was connected to a pressure transducer to continuously monitor the arterial blood pressure and heart rate, and the carotid artery catheter was used for shed blood withdrawal to induce pressure-controlled hemorrhagic shock. The jugular vein catheter was used to reinfuse the shed blood. The electrocardiogram was continuously recorded. After the rats were heparinized with intravenous heparin $(500 \mathrm{U} / \mathrm{kg})$, the shed blood was withdrawn into a heparinized syringe for a period of 10 minutes using a syringe pump and kept at room temperature. The mean blood pressure (MBP) was reduced and maintained at a target value of $\sim 30 \mathrm{mmHg}$ for 30 minutes. Shed blood volume was expressed as a percentage of calculated total blood volume (estimated from $6.12 \mathrm{~mL} / 100 \mathrm{~g}$ body weight; $\mathrm{Hu}$ et al., 2014). After 30 minutes of hypotension, the rats were resuscitated with the reinfusion of total shed blood for 30 minutes, and the rats were continuously monitored for another 30 minutes. At 1 hour after initiation of resuscitation, the catheters were removed from the femoral artery, carotid artery, and jugular vein, and the blood vessels tied off. The neck and groin incisions were closed in layers. The rats were returned to their cages to allow recovery from anesthesia. Postoperative analgesia (buprenorphine, $0.01 \mathrm{mg} / \mathrm{kg}$ body weight, subcutaneous) was maintained for 2 days. They were observed 6 weeks for survival.

\section{Experimental design}

Rats were randomized to $\mathrm{TH}(n=16)$ or normothermia group $(n=15)$. TH was started at 5 minutes after MBP reached $30 \mathrm{mmHg}$. The rats were placed into the ThermoSuit device and whole body surface was cooled by circulating ice water in accordance with the instructions for the ThermoSuit device (Life Recovery Systems, Kinnelon, NJ), which has been previously described in detail (Herring et al., 2015). Core temperature was initially reduced to $32^{\circ} \mathrm{C}$, and then the rats were taken out of the ThermoSuit device and placed on a heating pad. The body temperature continued to drop to $\sim 28^{\circ} \mathrm{C}$ followed by a gradual warm-up (Fig. 1) but stayed $<32^{\circ} \mathrm{C}$ until blood volume was fully restored. In the normothermia group, body temperature was maintained at $37^{\circ} \mathrm{C}$ during the procedure.

\section{Cardiac function}

Transthoracic echocardiographic imaging was performed at baseline before bleeding, 5 minutes before resuscitation with shed blood, and 1 hour after initiation of resuscitation, using a 15-MHz linear array transducer of a Philips ultrasound system. Echocardiograms were analyzed and measurements calculated post hoc in a blinded manner. Two-dimensional parasternal short-axis views and two-dimensional targeted M-mode tracings were taken to determine diastolic and systolic internal dimensions of the left ventricle (LVIDd and LVIDs, mm) and left ventricular fractional shortening (LVFS, $\%$, respectively.

\section{Blood gas analyses}

Blood samples of $0.3 \mathrm{~mL}$ were collected from the carotid arterial catheter at 1 hour after initiation of resuscitation. Blood $\mathrm{pH}$, arterial partial pressure of carbon dioxide $\left(\mathrm{PaCO}_{2}\right)$, arterial partial pressure of oxygen $\left(\mathrm{PaO}_{2}\right), \mathrm{Na}, \mathrm{K}, \mathrm{Cl}$, $\mathrm{iCa}$, glucose, lactate concentration, base excess (BE), hematocrit, and hemoglobin were measured with a blood gas analyzer. In

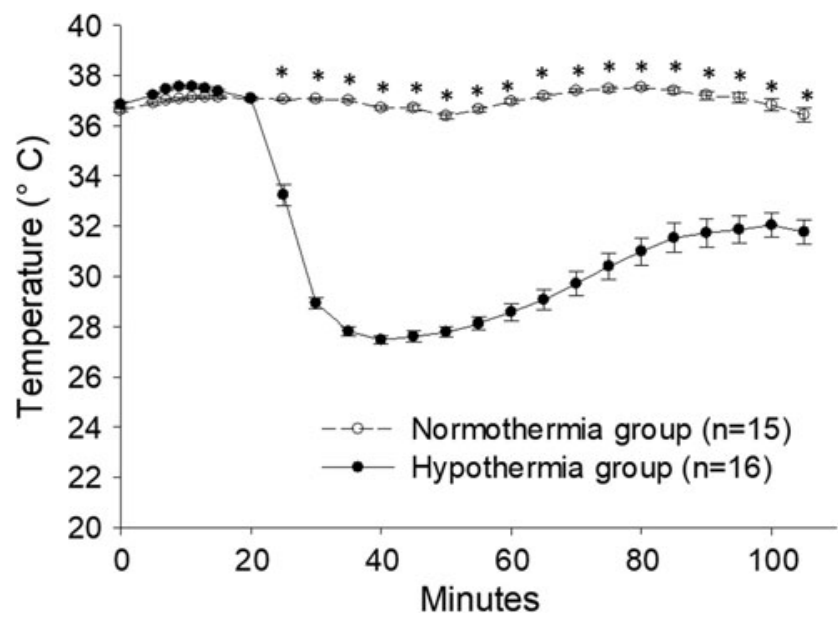

FIG. 1. The body temperature versus time. In the hypothermia group, temperature dropped to $28^{\circ} \mathrm{C}$ and then gradually increased to $\sim 32^{\circ} \mathrm{C}$ until blood volume was fully restored. The temperature remained $\sim 37^{\circ} \mathrm{C}$ in the normothermic group. ${ }^{*}$ Indicate those time points, at which there is significant difference between the two groups. 


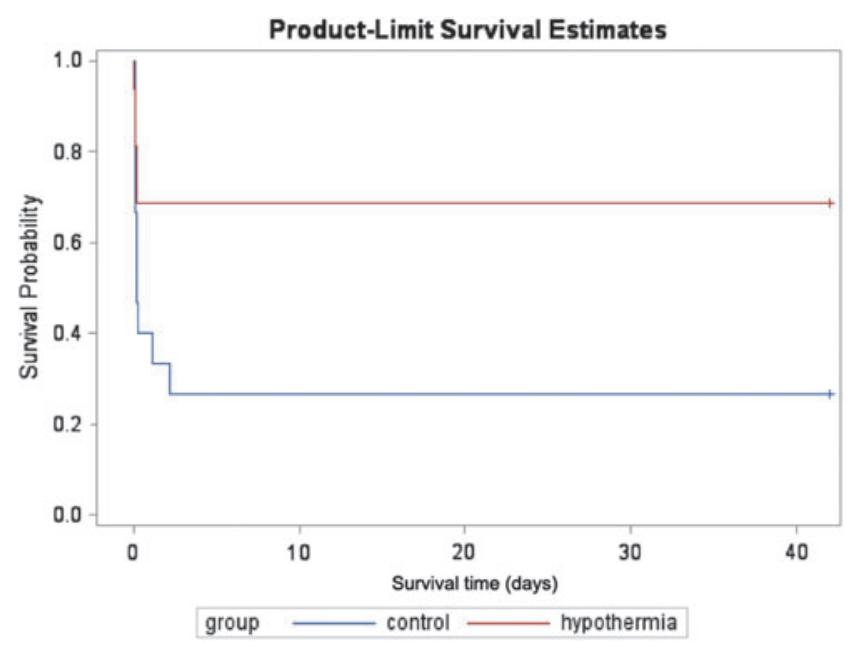

FIG. 2. The survival rate is significantly higher in the hypothermia group (red line) compared to control group (blue line; $p=0.04$, by Kaplan-Meier curve analysis). Rats that survived past 24 hours tended to survive for 6 weeks.

addition, blood was collected for additional cell counts, platelet count, and other electrolytes and chemistries at 1 hour after resuscitation.

\section{Tissue histological analysis}

At 6 weeks after hemorrhagic shock, all surviving rats were deeply anesthetized. To detect areas of microvascular damage in the tissues, thioflavin $\mathrm{S}$ was injected into the jugular vein. The rats were euthanized by an intravenous injection of $\mathrm{KCl}$.

After euthanasia, heart, brain, lungs, liver, and kidney were isolated, weighed, and photographed under white light, and under ultraviolet light to detect thioflavin $\mathrm{S}$ perfusion defects that demarcate microvascular obstruction. Triphenyltetrazolium chloride staining was used to identify necrosis (white areas) versus viable tissue (brick red areas). The tissues were fixed in $10 \%$ neutral buffered formalin and embedded in paraffin. The processed tissues were sectioned into $5-\mu \mathrm{m}$ slices and were used for hematoxylin and eosin $(\mathrm{H} \& \mathrm{E})$ staining for tissue structure, and picrosirius red staining to assess fibrosis.

\section{Statistical analyses}

Data are reported as mean \pm standard error of the mean. Student's $t$-tests were used for blood gas, blood chemistry, and echocardiographic results. A linear mixed-models analysis for repeated measures was used to analyze hemodynamic variables, testing for a group $\times$ time interaction. Differences between specific time points of hemodynamic variables were determined by post hoc contrasts from the same mixed models. Survival analysis was performed by log-rank testing. Values were considered significant at $p<0.05$.

\section{Results}

\section{Body temperature changes and shed blood volume}

Body temperature was comparable in hypothermic $\left(36.9^{\circ} \mathrm{C} \pm\right.$ $\left.0.1^{\circ} \mathrm{C}\right)$ and normothermic groups $\left(36.6^{\circ} \mathrm{C} \pm 0.1^{\circ} \mathrm{C}\right)$ at baseline. The changes in temperature in the $\mathrm{TH}$ and normothermic groups are shown in Figure 1 . The temperature remained $\sim 37^{\circ} \mathrm{C}$ during the surgical procedure in the normothermic group. In the $\mathrm{TH}$ group, temperature dropped to $28^{\circ} \mathrm{C}$ and then gradually rewarmed. During the shock phase, the total withdrawn blood volume (expressed as \% of estimated total blood volume) to maintain $\mathrm{MBP}$ at $30 \mathrm{mmHg}$ was similar in the TH group $(42.8 \% \pm 0.9 \%, n=16)$ and normothermic group $(44.5 \% \pm 1.4 \%, n=15 ; p=0.32)$.

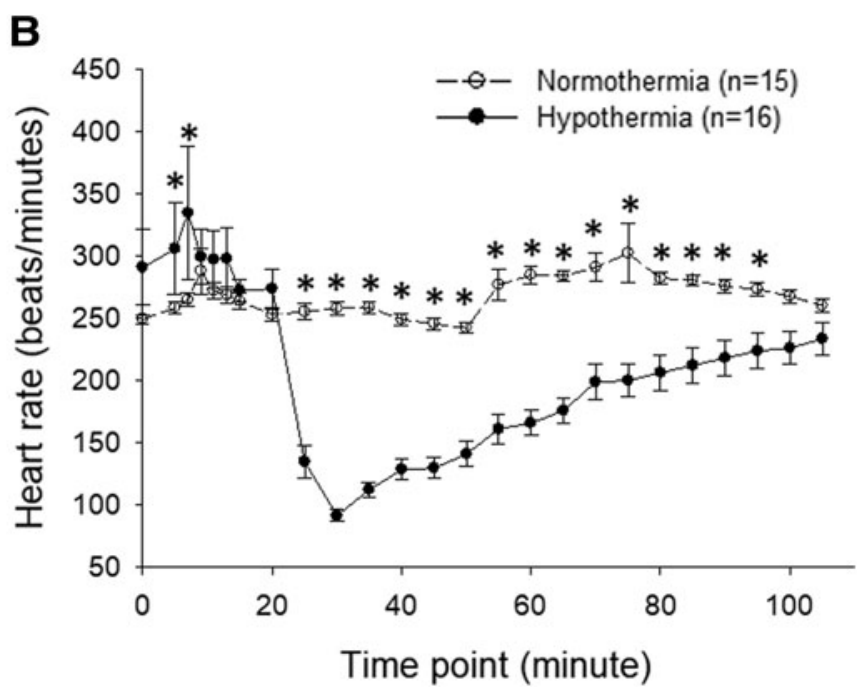

A

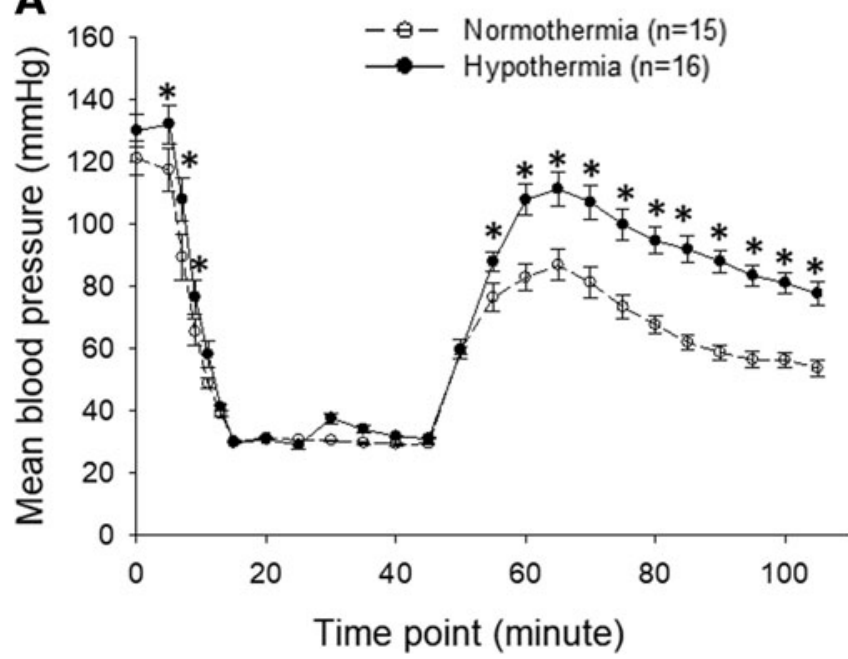

FIG. 3. MBP and heart rate. (A) Recovery of MBP during the resuscitation phase was significantly improved in the hypothermia group compared with the control group. [Group $\times$ time interaction term significant, $F(24,696)=3.18$, $p<0.0001] \mathrm{MBP}$ decreased gradually after initiation of blood withdrawing and maintained at $30 \mathrm{mmHg}$ during $30 \mathrm{minutes}$ of shock phase. Then the MBP rapidly increased after initiation of shed blood reinfusion. MBP increased in both groups, but significantly higher in the hypothermia group. (B) Heart rate was significantly lower in the hypothermia group compared with the control group during the procedure [Group $\times$ time interaction term significant, $F(24,696)=5.71, p<0.0001$ ] *Indicate those time points, at which there is significant difference between the two groups. MBP, mean blood pressure. 
TABle 1. Echocardiographic Results

\begin{tabular}{lllc}
\hline & $\begin{array}{c}\text { Normothermia } \\
(\mathrm{n}=15)\end{array}$ & TH $(\mathrm{n}=16)$ & $\mathrm{p}$ \\
\hline Baseline & & & \\
$\quad$ Diastolic ID (mm) & $6.33 \pm 0.13$ & $6.08 \pm 0.20$ & 0.331 \\
Systolic ID (mm) & $3.28 \pm 0.13$ & $3.23 \pm 0.16$ & 0.828 \\
LVFS (\%) & $48.3 \pm 1.4$ & $47.2 \pm 1.0$ & 0.552 \\
25 minutes of shock & & & \\
Diastolic ID (mm) & $2.71 \pm 0.15$ & $2.87 \pm 0.16$ & 0.479 \\
Systolic ID (mm) & $1.13 \pm 0.15$ & $0.15 \pm 0.04$ & $<\mathbf{0 . 0 0 0 1}$ \\
LVFS (\%) & $60.5 \pm 4.5$ & $94.5 \pm 1.3$ & $<\mathbf{0 . 0 0 0 1}$ \\
1 hour after blood & & & \\
reinfusion & & & \\
Diastolic ID (mm) & $4.88 \pm 0.22$ & $5.25 \pm 0.27$ & 0.308 \\
Systolic ID (mm) & $2.29 \pm 0.19$ & $1.81 \pm 0.23$ & 0.137 \\
LVFS (\%) & $53.9 \pm 3.3$ & $66.8 \pm 3.8$ & $\mathbf{0 . 0 1 8}$ \\
\hline
\end{tabular}

Bold values were significant at $p$ at least $<0.05$.

ID, internal dimension; LVFS, left ventricular fractional shortening; $\mathrm{TH}$, therapeutic hypothermia.

\section{Survival}

At 6 weeks, 4 of $15(26.7 \%)$ rats in the normothermic group and 11 of $16(68.8 \% ; p=0.032)$ rats in the TH group survived. Kaplan-Meier survival curves are shown in Figure 2. The curves show that $\mathrm{TH}$ significantly improved survival at the early stage and maintained this survival benefit up to 6 weeks analyzed by log-rank statistic $(p=0.04)$.

\section{$M B P$ and heart rate}

Recovery of MBP during the resuscitation phase was significantly improved in the $\mathrm{TH}$ group compared with the normothermic group (Fig. 3A). Heart rate, as expected, was significantly lower in the TH group compared with the normothermic group (Fig. 3B).

Table 2. Measures of Arterial Blood Gas PARAMETERS AND Electrolytes 1 Hour AfTER Resuscitation

\begin{tabular}{lccc}
\hline & Normothermia & TH & $\mathrm{p}$ \\
\hline $\mathrm{pH}$ & $7.43 \pm 0.01$ & $7.44 \pm 0.02$ & 0.589 \\
$\mathrm{pCO}_{2}(\mathrm{mmHg})$ & $22.9 \pm 1.9$ & $24.2 \pm 2.5$ & 0.680 \\
$\mathrm{pO}_{2}(\mathrm{mmHg})$ & $81.6 \pm 4.8$ & $117.8 \pm 9.9$ & $\mathbf{0 . 0 0 4}$ \\
$\mathrm{Na}(\mathrm{mmol} / \mathrm{L})$ & $134.1 \pm 0.9$ & $135.2 \pm 0.8$ & 0.309 \\
$\mathrm{~K}(\mathrm{mmol} / \mathrm{L})$ & $5.4 \pm 0.2$ & $4.1 \pm 0.1$ & $<\mathbf{0 . 0 0 0 1}$ \\
$\mathrm{Cl}(\mathrm{mmol} / \mathrm{L})$ & $112.3 \pm 0.6$ & $110.4 \pm 0.5$ & $\mathbf{0 . 0 2 4}$ \\
$\mathrm{iCa}(\mathrm{mmol} / \mathrm{L})$ & $1.2 \pm 0.01$ & $1.19 \pm 0.02$ & 0.918 \\
$\mathrm{Glu}(\mathrm{mg} / \mathrm{dL})$ & $336.8 \pm 17.9$ & $362.5 \pm 14.8$ & 0.331 \\
$\mathrm{Lac}(\mathrm{mmol} / \mathrm{L})$ & $1.8 \pm 0.2$ & $1.2 \pm 0.2$ & $\mathbf{0 . 0 2 8}$ \\
$\mathrm{Base}$ excess & $-6.82 \pm 0.5$ & $-5.56 \pm 0.3$ & $\mathbf{0 . 0 4 3}$ \\
$\quad(\mathrm{mmol} / \mathrm{L})$ & $35.8 \pm 0.8$ & $39.2 \pm 0.6$ & $\mathbf{0 . 0 0 2}$ \\
$\mathrm{Hct}(\%)$ & $11.9 \pm 0.3$ & $13.1 \pm 0.2$ & $\mathbf{0 . 0 0 3}$ \\
$\mathrm{Hb}(\mathrm{g} / \mathrm{dL})$ & & &
\end{tabular}

Bold values were significant at $p$ at least $<0.05$.

$\mathrm{pH}$, minus the log of the hydrogen ion $(\mathrm{H}+)$ concentration; $\mathrm{PaCO}_{2}$, arterial partial pressure of carbon dioxide; $\mathrm{PaO}_{2}$, arterial partial pressure of oxygen; $\mathrm{Na}$, sodium; $\mathrm{K}$, potassium; $\mathrm{Cl}$, chloride; $\mathrm{iCa}$, ionized calcium; Glu, glucose; Lac, lactate; Hct, hematocrit; Hb, hemoglobin.
Table 3. Blood Hematology and Biochemistry 1 Hour After Initiation of Shed Blood Reinfusion

\begin{tabular}{lccc}
\hline & Normothermia & TH & $\mathrm{p}$ \\
\hline Neutrophil $(\%)$ & $22.9 \pm 1.6$ & $16.1 \pm 1.4$ & $\mathbf{0 . 0 0 3}$ \\
Neutrophil $(/ \mu \mathrm{L})$ & $2223.7 \pm 248.7$ & $1756.6 \pm 219.4$ & 0.170 \\
Reticulocyte $(\%)$ & $2.61 \pm 0.3$ & $3.10 \pm 0.1$ & 0.180 \\
WBC $(\mathrm{K} / \mu \mathrm{L})$ & $9.67 \pm 0.7$ & $10.98 \pm 0.9$ & 0.257 \\
Absolute reticulocyte & $189.4 \pm 21.8$ & $227.5 \pm 11.9$ & 0.165 \\
$\quad(\mathrm{~K} / \mu \mathrm{L})$ & & & \\
RBC $(\mathrm{M} / \mu \mathrm{L})$ & $6.5 \pm 0.5$ & $6.3 \pm 0.6$ & 0.799 \\
Lymphocyte $(/ \mu \mathrm{L})$ & $7212.9 \pm 562.4$ & $8990.1 \pm 713.1$ & $\mathbf{0 . 0 6 1}$ \\
Lymphocytes $(\%)$ & $74.54 \pm 1.6$ & $81.7 \pm 1.4$ & $\mathbf{0 . 0 0 2}$ \\
Monocyte $(/ \mu \mathrm{L})$ & $120.3 \pm 30.4$ & $124.1 \pm 17.4$ & 0.914 \\
Monocytes $(\%)$ & $1.25 \pm 0.3$ & $1.16 \pm 0.2$ & 0.761 \\
Eosinophil $(/ \mu \mathrm{L})$ & $114.5 \pm 23.5$ & $108.5 \pm 10.7$ & 0.817 \\
Eosinophils $(\%)$ & $1.18 \pm 0.2$ & $1.1 \pm 0.1$ & 0.592 \\
MCV $(\mathrm{fL})$ & $63.5 \pm 0.7$ & $61.7 \pm 0.6$ & 0.066 \\
MCH $(\mathrm{pg})$ & $24.4 \pm 3.1$ & $25.3 \pm 3.4$ & 0.851 \\
MCHC $(\mathrm{g} / \mathrm{dL})$ & $38.2 \pm 4.5$ & $41.2 \pm 5.6$ & 0.685 \\
Platelet count $(\mathrm{K} / \mu \mathrm{L})$ & $556.0 \pm 39.0$ & $653.3 \pm 21.7$ & $\mathbf{0 . 0 6 1}$
\end{tabular}

Bold values were significant at $p$ at least $<0.05$.

$\mathrm{MCH}$, mean corpuscular hemoglobin; MCHC, mean corpuscular hemoglobin concentration; MCV, mean corpuscular volume; RBC, red blood cell; WBC, white blood cell.

\section{Cardiac function}

There were no differences in the echocardiographic measurements at baseline (Table 1). During the shock phase, systolic diameters were significantly smaller in the TH group. LVFS was markedly increased in the TH group compared with the normothermic group. At 1 hour after reinfusion of blood, LVFS in the TH group remained higher than in the normothermic group.

\section{Arterial blood analyses}

At 1 hour after resuscitation of hemorrhagic shock, $\mathrm{pO}_{2}$ (partial pressure of oxygen; $\mathrm{mmHg}$ ) remained essentially normal in TH $(117.8 \pm 9.9)$ but was reduced in the normothermic group $(81.6 \pm 4.8 ; p=0.004$; (Table 2$)$. The rats in the normothermia group had significantly elevated potassium, chloride, and lactate levels, and more negative BE compared with rats in the TH group. Platelet counts were preserved in TH group. Hematocrit and hemoglobin levels were greater in the hypothermia group (Table 2). There were no significant differences in $\mathrm{pH}, \mathrm{pCO}_{2}$, sodium, calcium, or glucose between the groups.

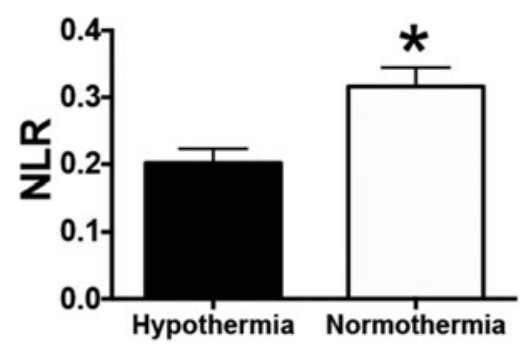

FIG. 4. The NLR at 1 hour after resuscitation. The NLR was significantly lower in hypothermia group versus the normothermia group. ${ }^{*} p<0.05$ versus hypothermia group. Data are expressed as mean \pm SEM. NLR, neutrophil-tolymphocyte ratio; SEM, standard error of the mean. 


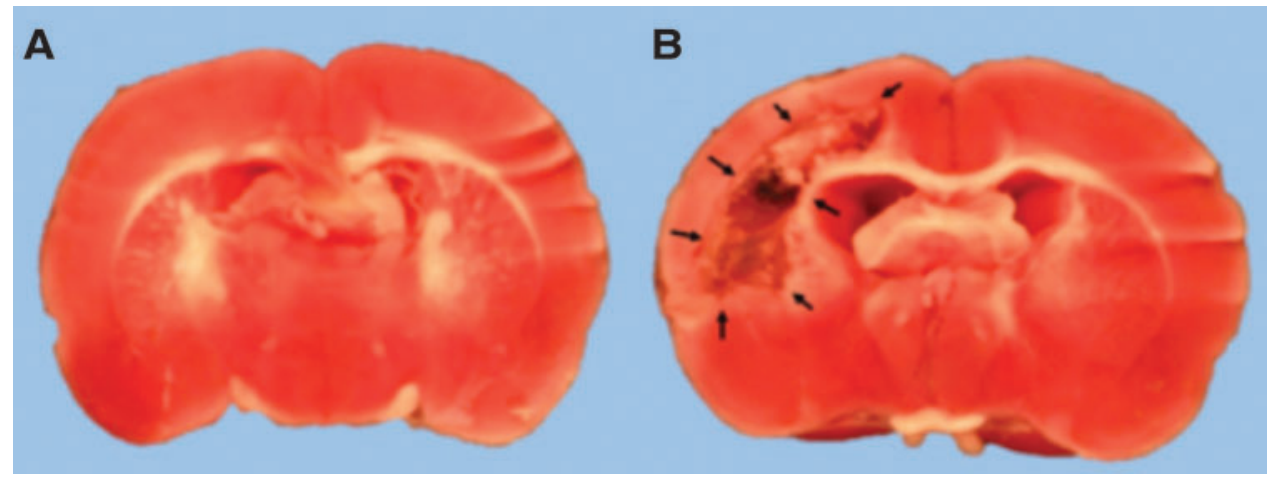

FIG. 5. Representative slices of brain stained with triphenyltetrazolium chloride.

(A) Hypothermia group;

(B) normothermia group. Note the large brain infarction shown in the normothermia sample. The black arrows outline the gross pathology of the cerebral infarction.

\section{Neutrophil-to-lymphocyte ratio}

The neutrophil-to-lymphocyte ratio (NLR) is a marker of inflammation associated with increased mortality in patients with severe hemorrhage shock. The NLR is calculated by dividing the absolute neutrophil count by the absolute lymphocyte count. The neutrophil count was lower in the TH group; the lymphocyte count was higher in the TH group (Table 3). The NLR was significantly lower in TH group $(0.20 \pm 0.02)$ compared with the normothermia group $(0.32 \pm$ $0.03 ; p=0.003$, Fig. 4).

\section{Pathological analysis}

At 6 weeks after hemorrhagic shock, gross pathology and light microscopy analyses were performed in the tissues from the surviving rats. Four of 15 rats survived in the normothermia group, and brain infarction was observed in 3 of the 4 surviving rats (75\%); 11 of 16 rats survived in the TH group, and brain infarction was observed in only 1 of the 11 surviving rats $(9 \%$; $p=0.033$; Fig. 5). H\&E-stained lung sections showed patchy lung lesions, including areas of thickened alveolar septa with mononuclear cell infiltration, disrupted alveolar morphology, and erythrocyte leaking into alveoli in the nomothermia and $\mathrm{TH}$ groups. There was no difference in lung pathology between groups. No evidence of myocardial, liver, or kidney infarction was detected by either gross pathology or by microscopic analysis. There were no architectural changes, inflammation, fibrosis, or necrosis in H\&E-stained heart, kidney, or liver sections in either group.

\section{Discussion}

The main finding of this study was that $\mathrm{TH}$ administered in the setting of hemorrhagic shock improved long-term survival, improved the recovery of MBP, and increased LVFS during the shock and resuscitation phases. TH blunted the inflammatory response of hemorrhagic shock as manifested by decreasing the NLR level. TH influenced the levels of blood gases and blood counts, favoring hypothermia over the control group.

Hypothermia, which occurs spontaneously in hemorrhagic shock patients, may be detrimental. Patients with severe hemorrhagic shock often become spontaneously hypothermic due to infusion of cold fluids, the use of anesthetic agents, and decreased ability to maintain normothermia. Spontaneous hypothermia is associated with poor outcomes that inhibit the coagulation pathway, reduce platelet func- tion, disrupt cellular homeostasis, and deplete energy stores (Kheirbek et al., 2009).

In contrast, $\mathrm{TH}$ can be beneficial; it protects organs from ischemic injury, improves cellular function and survival. The basic concept is that TH can decrease oxygen demand, preserve tissue ATP levels, and decrease metabolic activity. The results of our and other studies suggest that $\mathrm{TH}$ is a potent strategy for preserving organ function and improving survival during hemorrhage shock. In a sense, TH puts the body into a state of suspended animation. Prueckner et al. (2001) tested rats that underwent pressure-controlled hemorrhagic shock at a mean arterial blood pressure (MAP) of $40 \mathrm{mmHg}$ for 90 minutes followed by resuscitation. They found that brief mild hypothermia had a physiological benefit and tended to improve survival; prolonged mild hypothermia significantly increased survival after severe hemorrhagic shock. Takasu et al. $(2000,2006)$ found that TH prolonged the survival time in rats during uncontrolled hemorrhagic shock, increased the blood pressure, and decreased the heart rate, without affecting the respiratory rate. Furthermore, swine hemorrhagic shock models confirmed that TH improved survival, preserved organ viability and cognitive function (Takasu et al., 2003). These studies assessed short-term survival only, from a few hours to 24 hours. Our study assessed the long-term survival at 6 weeks. We found that TH improves the long-term survival in rats subjected to hemorrhagic shock.

Mild $\left(32^{\circ} \mathrm{C}\right.$ to $\left.35^{\circ} \mathrm{C}\right)$, moderate $\left(28^{\circ} \mathrm{C}\right.$ to $\left.32^{\circ} \mathrm{C}\right)$, or deep $\left(<28^{\circ} \mathrm{C}\right)$ induced hypothermia is used as a protective strategy for the brain and vital organs during cardiopulmonary bypass for cardiac surgery (Engelman et al., 2015). In our study, core temperature was initially reduced to $32^{\circ} \mathrm{C}$, and then the rats were taken out of the ThermoSuit device and placed on a heating pad. The body temperature continued to drop to $\sim 28^{\circ} \mathrm{C}$ during a brief time (about 20 minutes) followed by a gradual warm-up. Of note, previous studies have examined the outcome of temperatures of $28^{\circ} \mathrm{C}$ or less versus more mild hypothermia of $35^{\circ} \mathrm{C}$ in human subjects undergoing cardiopulmonary bypass for cardiac surgery. In one study by Martin et al. (1994), temperatures of $28^{\circ} \mathrm{C}$ or lower were associated with a lower incidence of neurological morbidity than temperatures of $35^{\circ} \mathrm{C}$ or higher. In another study by Mora et al. (1996), the effect of cardiopulmonary bypass temperatures on central nervous systems outcomes were assessed in patients who were cooled to a temperature $<28^{\circ} \mathrm{C}$ versus temperatures of at least $35^{\circ} \mathrm{C}$. They found that there was a significantly lower incidence of perioperative neurological risk in those patients who received the $28^{\circ} \mathrm{C}$ of $\mathrm{TH}$ versus 
those who had a minimum temperature of $35^{\circ} \mathrm{C}$. Thus, $28^{\circ} \mathrm{C}$ appeared safe and efficacious in these clinical studies. In addition, in our study, the $28^{\circ} \mathrm{C}$ was associated with a higher long-term survival rate and other biochemical parameters favoring organ function.

Hemorrhagic shock has been shown to induce an inflammatory response. Coyan et al. (2014) reported that hypothermia significantly attenuated leukocyte adherence and mast cell degranulation in a pressure-controlled hemorrhagic shock rat model. They found that decreased microvascular inflammation persisted and the effect carried over after the $\mathrm{TH}$ period. In a swine model of lethal vascular injuries (Chen et al., 2005), profound hypothermia modulated the postshock immune response by attenuating proinflammatory IL-6, increasing antiinflammatory IL-10, and augmenting the expression of protective HSP-70 protein. Recently, we observed that $\mathrm{TH}$ downregulated the expression of proinflammatory genes (Shi et al., 2017).

The NLR has been identified as an independent predictor of mortality in spontaneous intracerebral hemorrhage. Compared with conventional cytokine-related inflammatory biomarkers, NLR is more easily accessed from blood cell counts and accurately reflects inflammatory status. Lattanzi et al. (2016) demonstrated that in patients with acute intracerebral hemorrhage, higher neutrophils, lower lymphocytes, and higher NLRs predicted worse 3-month outcome. A retrospective cohort study of 566 trauma patients (Soulaiman et al., 2020) revealed that increased NLR was associated with higher in-hospital mortality. Elevated NLR on day 1 has high predictive power for overall survival during the first 30 days after trauma. Our finding is in line with these studies and demonstrates that the inflammatory response occurs very quickly (1 hour after resuscitation) after hemorrhagic shock. Hypothermia inhibited the inflammation as shown by a reduction in the NLR level. NLR level could be an easily obtained early marker of the success of therapies to treat hemorrhagic shock, including TH.

Arterial blood gas analysis is commonly performed in hemorrhage shock patients to evaluate early acid-base disturbances and provide valuable information regarding perfusion and oxygenation. The most commonly used parameters are serum lactate and $\mathrm{BE}$, which provide information about the degree of anaerobic metabolism. Lactate, a product of anaerobic glycolysis, has been used to determine the severity of tissue hypoperfusion in hemorrhagic shock. Multiple studies have demonstrated that increased lactate is an important predictor of morbidity and mortality in hemorrhage shock. Abramson et al. (1993) documented 100\% survival when normalization of serum lactate occurred within the first 24 hours, $78 \%$ survival if normalization occurred in 24-48 hours, and only $14 \%$ survival if normalization did not occur within 48 hours. Large retrospective studies found that both high initial levels of lactate as well as poor lactate clearance were strong predictors of mortality in trauma patients (Regnier et al., 2012; Odom et al., 2013).

$\mathrm{BE}$ is a calculated measure of the amount of sodium bicarbonate and $\mathrm{pH}$ of the blood. Rixen and Siegel (2005) found that $\mathrm{BE}$ values were an indicator of severity of hemorrhagic shock. A study from the Keel group found that BE predicts mortality in patients with severe hemorrhagic shock (Abt et al., 2009). Davis et al. (1991) also demonstrated that BE could remain abnormal despite postresuscitation improvements in MAP and $\mathrm{O}_{2}$ saturation in an animal model of hemorrhagic shock. Our study indicated that the TH decreased lactate and $\mathrm{BE}$ value; $\mathrm{pO}_{2}$ and platelet counts were preserved in $\mathrm{TH}$ group. We also observed that hematocrit and hemoglobin level were greater in the TH rats. The result may suggest cooling-induced hemoconcentration (Norio et al., 2002).

TH was observed to possess a positive inotropic effect in this study, which may be related to the improved postresuscitation survival rate. The underlying mechanisms need to be further investigated. Hypothermia may increase the cardiac contractility by enhancing phospholamban phosphorylation leading to enhanced SERCA-related $\mathrm{Ca}_{2}{ }^{+}$uptake by sarcoplasmic reticulum with stronger excitation/contraction coupling of cardiac cells (Li et al., 2019). Another possible mechanism is that hypothermia may increase $\mathrm{Ca}_{2}+$ sensitivity of myofilament proteins and improve $\mathrm{Ca}_{2}{ }^{+}$-activated force generation (Kusuoka et al., 1991; Stowe et al., 1999)

\section{Conclusion}

TH administered in the setting of hemorrhagic shock improved long-term survival, blunted the inflammatory response, and preserved aspects of blood gases and blood chemistries. This finding could be applied to survival after not only hemorrhage in the battle field, but could be applied to civilians after car accidents or gunshot wounds, or other causes of bleeding.

\section{Author Disclosure Statement}

No competing financial interests exist.

\section{Funding Information}

Dr. R.A.K. is the principal investigator of this study, and the project was supported by the Office of the Assistant Secretary of Defense for Health Affairs, through the Peer Reviewed Medical Research Program under Award No.W81XWH-16-10606.

\section{References}

Abramson D, Scalea TM, Hitchcock R, et al. Lactate clearance and survival following injury. J Trauma 1993;35:584-588.

Abt R, Lustenberger T, Stover JF, et al. Base excess determined within one hour of admission predicts mortality in patients with severe pelvic fractures and severe hemorrhagic shock. Eur J Trauma Emerg Surg 2009;35:429.

Chen Z, Chen H, Rhee P, et al. Induction of profound hypothermia modulates the immune/inflammatory response in a swine model of lethal hemorrhage. Resuscitation 2005;66: 209-216.

Coyan GN, Moncure M, Thomas JH, et al. Induced hypothermia during resuscitation from hemorrhagic shock attenuates microvascular inflammation in the rat mesenteric microcirculation. Shock 2014;42:518-524.

Dai W, Herring MJ, Hale SL, et al. Rapid surface cooling by ThermoSuit system dramatically reduces scar size, prevents post-infarction adverse left ventricular remodeling, and improves cardiac function in rats. J Am Heart Assoc 2015;4:e002265.

Dai W, Shi J, Carreno J, et al. Improved long-term survival with remote limb ischemic preconditioning in a rat fixed-pressure hemorrhagic shock model. Cardiovasc Drugs Ther 2019;33: 139-147. 
Davis JW, Shackford SR, Holbrook TL. Base deficit as a sensitive indicator of compensated shock and tissue oxygen utilization. Surg Gynecol Obstet 1991;173:473-476.

Eastridge BJ, Holcomb JB, Shackelford S. Outcomes of traumatic hemorrhagic shock and the epidemiology of preventable death from injury. Transfusion 2019;59:1423-1428.

Engelman R, Baker RA, Likosky DS, et al. The Society of Thoracic Surgeons, The Society of Cardiovascular Anesthesiologists, and The American Society of ExtraCorporeal Technology: clinical Practice Guidelines for Cardiopulmonary Bypass-Temperature Management during Cardiopulmonary Bypass. J Extra Corpor Technol 2015;47:145-154.

Hale SL, Kloner RA. Myocardial temperature in acute myocardial infarction: protection with mild regional hypothermia. Am J Physiol 1997;273:H220-H227.

Hale SL, Kloner RA. Mild hypothermia as a cardioprotective approach for acute myocardial infarction: laboratory to clinical application. J Cardiovasc Pharmacol Ther 2011;16:131-139.

Herring MJ, Dai W, Hale SL, et al. Rapid induction of hypothermia by the ThermoSuit system profoundly reduces infarct size and anatomic zone of no reflow following ischemiareperfusion in rabbit and rat hearts. J Cardiovasc Pharmacol Ther 2015;20:193-202.

Herring MJ, Hale SL, Dai W, et al. Hypothermia in the setting of experimental acute myocardial infarction: a comprehensive review. Ther Hypothermia Temp Manag 2014;4:159-167.

Hooper TJ, Nadler R, Badloe J, et al. Implementation and execution of military forward resuscitation programs. Shock 2014;41 Suppl 1:90-97.

$\mathrm{Hu} \mathrm{X}$, Yang Z, Yang M, et al. Remote ischemic preconditioning mitigates myocardial and neurological dysfunction via K(ATP) channel activation in a rat model of hemorrhagic shock. Shock 2014;42:228-233.

Kalkwarf KJ, Cotton BA. Resuscitation for hypovolemic shock. Surg Clin North Am 2017;97:1307-1321.

Kalogeris T, Baines CP, Krenz M, et al. Cell biology of ischemia/reperfusion injury. Int Rev Cell Mol Biol 2012;298: 229-317.

Kheirbek T, Kochanek AR, Alam HB. Hypothermia in bleeding trauma: a friend or a foe? Scand J Trauma Resusc Emerg Med 2009; 17:65.

Kusuoka H, Ikoma Y, Futaki S, et al. Positive inotropism in hypothermia partially depends on an increase in maximal $\mathrm{Ca}(2+)$-activated force. Am J Physiol 1991;261:H1005-H1010.

Lattanzi S, Cagnetti C, Provinciali L, et al. Neutrophil-tolymphocyte ratio predicts the outcome of acute intracerebral hemorrhage. Stroke 2016;47:1654-1657.

Li J, Zhu X, Wang H, et al. Akt1-mediated CPR cooling protection targets regulators of metabolism, inflammation and contractile function in mouse cardiac arrest. PLoS One 2019; 14:e0220604.

Martin TD, Craver JM, Gott JP, et al. Prospective, randomized trial of retrograde warm blood cardioplegia: myocardial benefit and neurologic threat. Ann Thorac Surg 1994;57:298-302.
Mora CT, Henson MB, Weintraub WS, et al. The effect of temperature management during cardiopulmonary bypass on neurologic and neuropsychologic outcomes in patients undergoing coronary revascularization. J Thorac Cardiovasc Surg 1996;112:514-522.

Norio H, Takasu A, Kawakami M, et al. Rapid body cooling by cold fluid infusion prolongs survival time during uncontrolled hemorrhagic shock in pigs. J Trauma 2002;52:1056-1061.

Odom SR, Howell MD, Silva GS, et al. Lactate clearance as a predictor of mortality in trauma patients. J Trauma Acute Care Surg 2013;74:999-1004.

Prueckner S, Safar P, Kentner R, et al. Mild hypothermia increases survival from severe pressure-controlled hemorrhagic shock in rats. J Trauma 2001;50:253-262.

Regnier MA, Raux M, Le Manach Y, et al. Prognostic significance of blood lactate and lactate clearance in trauma patients. Anesthesiology 2012;117:1276-1288.

Rixen D, Siegel JH. Bench-to-bedside review: oxygen debt and its metabolic correlates as quantifiers of the severity of hemorrhagic and post-traumatic shock. Crit Care 2005;9: 441-453.

Shi J, Dai W, Kloner RA. Therapeutic hypothermia reduces the inflammatory response following ischemia/reperfusion injury in rat hearts. Ther Hypothermia Temp Manag 2017;7:162170.

Soulaiman SE, Dopa D, Raad AT, et al. Cohort retrospective study: the neutrophil to lymphocyte ratio as an independent predictor of outcomes at the presentation of the multi-trauma patient. Int J Emerg Med 2020;13:5.

Stowe DF, Fujita S, An J, et al. Modulation of myocardial function and $\left[\mathrm{Ca}_{2}{ }^{+}\right]$sensitivity by moderate hypothermia in guinea pig isolated hearts. Am J Physiol 1999;277:H2321$\mathrm{H} 2332$.

Takasu A, Norio H, Gotoh Y, et al. Effect of inducedhypothermia on short-term survival after volume-controlled hemorrhage in pigs. Resuscitation 2003;56:319-328.

Takasu A, Sakamoto T, Okada Y. Effect of induction rate for mild hypothermia on survival time during uncontrolled hemorrhagic shock in rats. J Trauma 2006;61:1330-1335.

Takasu A, Stezoski SW, Stezoski J, et al. Mild or moderate hypothermia, but not increased oxygen breathing, increases long-term survival after uncontrolled hemorrhagic shock in rats. Crit Care Med 2000;28:2465-2474.

Address correspondence to: Jianru Shi, PhD

HMRI Cardiovascular Research Institute Huntington Medical Research Institutes 686 S. Fair Oaks Avenue Pasadena, CA 91105

USA

E-mail: jianru.shi@hmri.org 